Volume 12, Issue 4, pp. 045 - 57

Article Info

Accepted: 27/11/2021

Corresponding Author: $\left({ }^{*}\right)$ geod17010@geo.aegean.gr

DOI: https://doi.org/10.48088/ejg.p.ago.12.4.045.057

Research Article

\title{
Spatial Analysis on Networks: Towards identifying similarity of routes
}

\author{
Panagiotis Agourogiannis ${ }^{1^{*}}$, \\ (1) Dimitris Kavroudakis ${ }^{1}$ \& \\ (1) Marios Batsaris ${ }^{1}$ \\ ${ }^{1}$ University of the Aegean, Greece
}

\section{Keywords}

Network Analysis,

Spatial Networks,

Points of Interest,

Spatial Similarity,

GIS,

Spatial Analysis

\begin{abstract}
Finding an optimal path in a road network is a method of planning and decision-making that is mainly related to transportations and emergency response. The paper presents an algorithm for finding optimal paths in spatial networks, through the utilization of open source GIS and mathematical analysis of Networks using Graph Theory as well as using geographical proximity attributes of network nodes. The geometric and spatial information of the network as well as its relations with points of interest (POI) of the study areas located at the nodes and edges of the network, are transformed into spatial information, which by applying spatial queries in a geographical database (Postgis/Pgrouting) give query-enabled paths. The case study for the application of the algorithm and finding a route based on spatial queries is the island of Lesvos. This island combines intense topography and a complex road network with multiple geometric relationships. The area also has points of interest such as cultural, tourist and social. The final route will be a synthesis of morphological, tourist and cultural elements similar to the spatial search queries. Finally, the methodology as well as the search algorithm can be applied to any Spatial Network (transportations, environment, energy) described by its geographical features, considering all kinds of geographical issues, thus solving spatial problems and contributing to local development.
\end{abstract}

\section{Highlights:}

-Search algorithm to find paths in spatial network using GIS and Graph Theory.

-Decision making about local development using spatial networks analysis.

-Network analysis to find similar paths with the same spatial characteristics. 


\section{INTRODUCTION}

A spatial network can be represented as a mathematical graph and analyzed on the basis of its topological features. This mathematical representation uses nodes and edges to represent road segments and road junctions. Nodes are the mathematical representation of intersections and edges are the mathematical representation of the road segments. However, although this abstract representation method (mathematical network analysis) solves the problem of analyzing a complex spatial network, giving it a simplified and coherent form, the network lack information related to location attributes and with the relationships which satisfy the principles of The First Law of Geography (W. Tobler, 1970). For example, for the analysis of a road network, characteristics such as elevation differences are important factors however, they cannot be captured directly by the network in the form of a mathematical graph.

Using spatial analysis approaches, we can produce informative graphical representation of a spatial network. This network includes not only geometrical attributes, but other geographyrelated attributes such as altitude, slope, aspect to name a few.

Typical algorithms such as Dijkstra's shortest path (E.W. Dijkstra, 1959) and "Travelling Salesman Problem" are based on the spatial network topology focusing on features that address the cost of moving between nodes, expressed either in units of distance or time.

Understanding the nature of networks and realizing that in order to solve spatial problems, we must represent a network accurately, the need arises to develop tools (tools for spatial analysis of the geographical data of a network and the spatial area in which it is located) that combine both Graph Theory and Geographic Information Systems (GIS) approaches. In this way, the information in the spatial network, such as the terrain altitude and the distance from POls (Points Of Interest), will be able to be integrated in the structural elements of the graph, and then used for spatial queries related a number of scientific disciplines such as urban planning, athletic events and spatial optimization (C. Yu et al, 2003).

In the relevant scientific literature a multitude of works focus on spatial network analysis combining the theory of Graphs and types of existing network algorithms with GIS (W. Zeng et al, 2009; H. Hu et al, 2016; Ahmadzai, F. et al, 2019). The study of B. Lu et al. (2018) developed an algorithm for the conversion of a spatial network into a graph through geoinformatics tools considering shortest routes in an urban area.

GIS are an integrated tool that is useful and supportive towards efficient policy-making and decision-making through the management of large volumes of spatial data (Malczewski, 2006; Fischer, M.M. and Nijkamp, P., 1992) such as spatial networks. Their contribution is reported in studies, describing identification of shortest paths in a road network for emergency planning (Derekenaris et al., 2001; Panahi, S. et al, 2008; Ahmed et al., 2017; Chen, Q. et al., 2019) and in particular in cases of traffic congestion (Elsheikh et al, 2016) as well as in the optimal location of a fuel station based on spatial network analysis (Kuby et al., 2007). Also, Feldman et al. (1995) utilized least-cost paths for routing oil pipelines near the Caspian Sea using GIS and remote sensing data and methods. Finally, the study of Shuo Shang et al. (2016) accelerated the shortest path computing in dynamic spatial networks, creating mobile applications, such as route planning and recommendation, car navigation and tracking, and location-based services in general.

The number of applications and studies about spatial network analysis cover a wide range of topics. In the field of transport network-related analysis focus on travelling distance, correlation between population accessibility and road network (Paraskevopoulos et al, 2019), navigation, routing and traveling time, based on actual network drive time (Fischer, M.M., 2006). In transportation-related network analysis, proximity to relevant geographical features is not included so far and objects are evaluated mainly as part of the network. This work presents a novel way of incorporating geographical features that are not actually "on" the network but can be used to characterize parts of the network such as edges and nodes. The proposed geographical attributes will "enrich" the network with additional geography-related information, in order to be used for optimal path finding for: athletic events, for running and training as well as possible historical walks and other cultural events. Thus the present work 
fills a scientific gap in the international literature on the use of spatial network analysis for identifying similarity of routes. More specific this work fills the gap of using not only geometric (topology) aspects of a spatial network but also the geographical characteristics (proximity to POls) of spatial networks.

In particular, the analysis of spatial networks for athletic events is strongly related to finding optimal training routes for preparation and participation in major sporting events. To our knowledge, there is a need to find routes that will simulate the race route to be used for training by the general public and / or by high-performance athletes. With this approach athletes can identify a suitable training route close to their place of residence for daily training without need to travel long distances to the target area. This will help them during the preparation for the athletic event and help them achieve better results.

In this article, we will present an algorithm for finding paths in a road network, including additional variables, such as altitude and distance from POls, while maintaining all topological properties of the Graph. The main purpose of analyzing a road network is to obtain a final acceptable route that will be as similar as possible to a target path from another network. It will include spatial criteria that will be used in the search algorithm. The spatial criteria will refer to the length of the route, the altitude difference and the distance from points of interest. In other words, the proposed algorithm will use geographical proximity to POls and other related features, which are "outside" the graph, to characterize nodes and edges of the spatial graph.

The process of converting the spatial network into a graph as well as calculating the geographical and metric relationships that were recorded as attribute values at nodes and edges, was accomplished using the PostgreSQL database management system and the PostGIS (2019) and pgrouting (2019) tools for spatial analysis and routing respectively. The algorithm was developed in the programming language $R$ (CRAN, 2019) which is an opensource programming language and it is an implementation of the $S$ language for statistical computing and graphics (Becker et al., 1988). $R$ language provides a wide variety of statistical packages and graphical techniques for analyzing spatial data and presenting results on charts and maps. One of its strength is the ability to communicate with other open-source geoinformatics software and geographic database management systems. This is quite useful, especially when analyzing large spatial networks in tabular format (nodes, edges).

The proposed method is suitable for a wide variety of networks. This may include roadnetwork, hydrological-network, electricity-networks as well as social networks. The very same principles can be used across a number of disciplines using network formatted datasets.

\section{METHODOLOGY \& DATA}

The mathematical analysis of spatial networks and the management of big geographical data presupposes the systematic and mathematically standardized methodology that focuses on the definition of hierarchical analysis steps. This part of the paper describes the sequential steps for finding a route in a spatial network combining graph theory as well as spatial analysis, and geographical databases.

Using a Spatial Database system is necessary in this type of analysis as network structures are quite complex especially when associated and linked with geographical objects on/off the network. Specifically, we use the Postgresql database along with the Postgis/Pgrouting plugin which enables storage and analysis of spatial objects in the database. For demonstration purposes, we used Lesvos Island in Greece in order to illustrate the algorithm potentials.

The spatial network used consists of a well-curated road network of the island, which comes from the "Open Street Map" (OSM) open spatial data platform. This is a set of 189661 nodes and 192587 edges representing the road network of the island. It has been corrected over a number of possible geometry faults such as loops, and is now routable. From the "Open Street Map" came the spatial data of the points of interest that represent the settlements, the cultural monuments and the tourism enterprises of the study area. Points of interest are an important 
factor in network analysis as they are used as criteria to characterize the finding route. Finally used Digital elevatio model (DEM) spatial resolution 25 meter, which comes from the European Union's Earth observation programme Copernicus and all information is free and openly accessible to all users. All spatial data, vector (road network, POI) and raster (DEM), transformed from the global reference system(WGS84) to the Greek projection system(Greek grid), ensuring the same metric system and scale. We used the R programming language for data analysis.

After querying the Spatial Database for routes based only on total length, then we start filtering the results in R with the use of the "dplyr" R package (2021). The query results returned a set of network paths with edges and vertices. Looping over this set, we calculated statistical aspects for each possible path based on the altitude (starting and finishing node), the aspect and the proximity of the path to POI. Now, the Spatial Database is ready for specialized queries regarding paths in the roads of the island. The queries can include not only the distance of the path but also the additional attributes we have calculated such as for example:

"Find all paths in the island with a total distance from $40 \mathrm{~km}$ to $41 \mathrm{~km}$, that end near a big city and pass very close to Archeological Sites."

or/and

"Find all paths in the island that start from a village and pass very close to hotels but far away from industrial complexes"

This type of query couldn't be used earlier as the Spatial Database required information about POI and characteristics of road segments. To further illustrate our innovative approach we use a real case study that highlights the potential strengths of our approach and its use in athletic event training. The steps of the methodology for identifying a suitable network path are the following:

Step 1: Preparation of the spatial network. As the first part of the proposed methodology the road network is automatically "split" into smaller sections depending on the length of the path to be found. The process of shaping the road network is considered necessary to identify the desired length of routes in an area. For example, to identify $10-20 \mathrm{~km}$ routes, the road is divided into multiple $500 \mathrm{~m}$ sections. For longer routes these sections may have a length of $1,000 \mathrm{~m}$. Next, the intersection points (nodes) are determined. This stage is performed only once for each area and does not need to be repeated, except in the respective updated version of the road network of the study area. The disassembly of spatial data in tabular format of nodes and edges along with the creation of an adjacency matrix in the Postgresql database hold connections between geometrical features. The result of this step includes a number of tables and a routing table of our spatial network for further analysis.

Step 2: Inserting spatial information in the node and edge tables of the spatial network. Altitude characteristics of the study area is an important spatial parameter which can influence the results considerably. We draw on information about altitude and slope of the network, giving the possibility to study the minimum, maximum and average altitude of the network paths. In this step we assign altitude information to each node of the spatial network. Topography is a very important parameter for the spatial resolution of the spatial network as mountains and valleys have strong influence on the structure of spatial networks, in cases such as the cost and feasibility of transportation projects (Rodrigue, 2013). For the case study of athletic events, we need altitude information in order to choose a network path for training that include specific quantities of up-hill and down-hill paths which increase the difficulty level of the athletic event and require specialized strategy from the athletes in terms of strength and stamina.

Step 3: Distance between POIs and network nodes. In this step, we calculate the distance of each node of the spatial network from the POls of the study area. The POls are important in our results as they fulfill basic criteria for charting paths. However, the importance of the 
POI differs, depending on the network problem we have to solve for example: cultural routes, athletic routes or scenic routes (Agourogiannis et al, 2019). For this reason, every time we execute this step of the methodology, we use different weights according to the context of the network problem. Finally, we create a matrix with all values describing the node of the spatial network of the study area. This step consumes a substantial amount of computational power of the system which is directly proportional to the amount of POI categories as well as their spatial distribution in the study area.

Step 4: Recursive Search. The fourth step of the proposed algorithm includes a recursive search approach. In more detail, we expand the typical data base search process by using spatial information in the table that includes all the spatial features and attributes of the road network. The goal is to identify similar routes in the network based on predefined "similarity" criteria. This part is computationally expensive as it requires numerous spatial calculations. Finally data export is done in vector form $\left({ }^{*} . \mathrm{shp},{ }^{*} . \mathrm{gpx},{ }^{*} . \mathrm{kmz},{ }^{*}\right.$.csv) for use in various dataformats and further visualization by stakeholders.

\section{CASE STUDY \& RESULTS}

This part presents the use of a method which can find spatial paths in a road network through the use of GIS and spatial analysis methods.

A spatial search algorithm has been developed for the identification of paths on a network. This search algorithm identifyies paths that fulfil a set of criteria based on similarity. In other words, it returns a set of similar paths based on a number of criteria.

We have used this "search algorithm" in order to identify similar sport-related routes (such as a marathon event) in the road network of Lesvos island, Greece. The novelty of our approach in the use of "proximity" to POls that has been pre-calculated for all nodes of our road network.

The algorithm developed is based on spatial "filters" applied to the road network and calculates geometric characteristics of the network (distance, altitude etc.). Also, we used filters to describe the POI such as sites of historical, cultural and environmental value and settlements.

The study area is the island of Lesvos as it includes a wide variety of landscapes in terms of topographic characteristics. Its road network consists of a number of sub-networks developed under different decades and spanning across 4087,61 km. Finally, in this region there are many points of interest that contribute to the local development and promotion, such as traditional settlements, museums, archaeological and religious sites (table 1). POls selection of this case study is based on a touristic athletic event that includes information about a number of cultural and touristic points on the island of Lesvos. The selection of POIs varies according to the thematic area of the case study, reflecting possible attraction and repelling factors of the suitability of the network path.

Table 1. Spatial data used in spatial network analysis

Title

Lesvos Road Network
Lesvos DEM
Lesvos Settlements
Lesvos Cultural sites

Lesvos Tourism business
Format

Spatial

Spatial

Spatial

Spatial

Spatial
Source

OSM

Copernicus

OSM

OSM

OSM 
Following the steps described in the methodology and the transformation of the network into a Graph (Figure 1), the metric relations between the network nodes were calculated, as well as the geographical characteristics of the study area and the points of interest.

The resulting spatial information was stored in a spatially enriched database using Postgresql (table 2, 3). As can be seen in the following Figure 1, the road network includes a very dense nodes point pattern. This enables the accurate depiction of the actual 3D representation of the network as well as it helps towards the detailed "spatial search" of any possible path in this network.

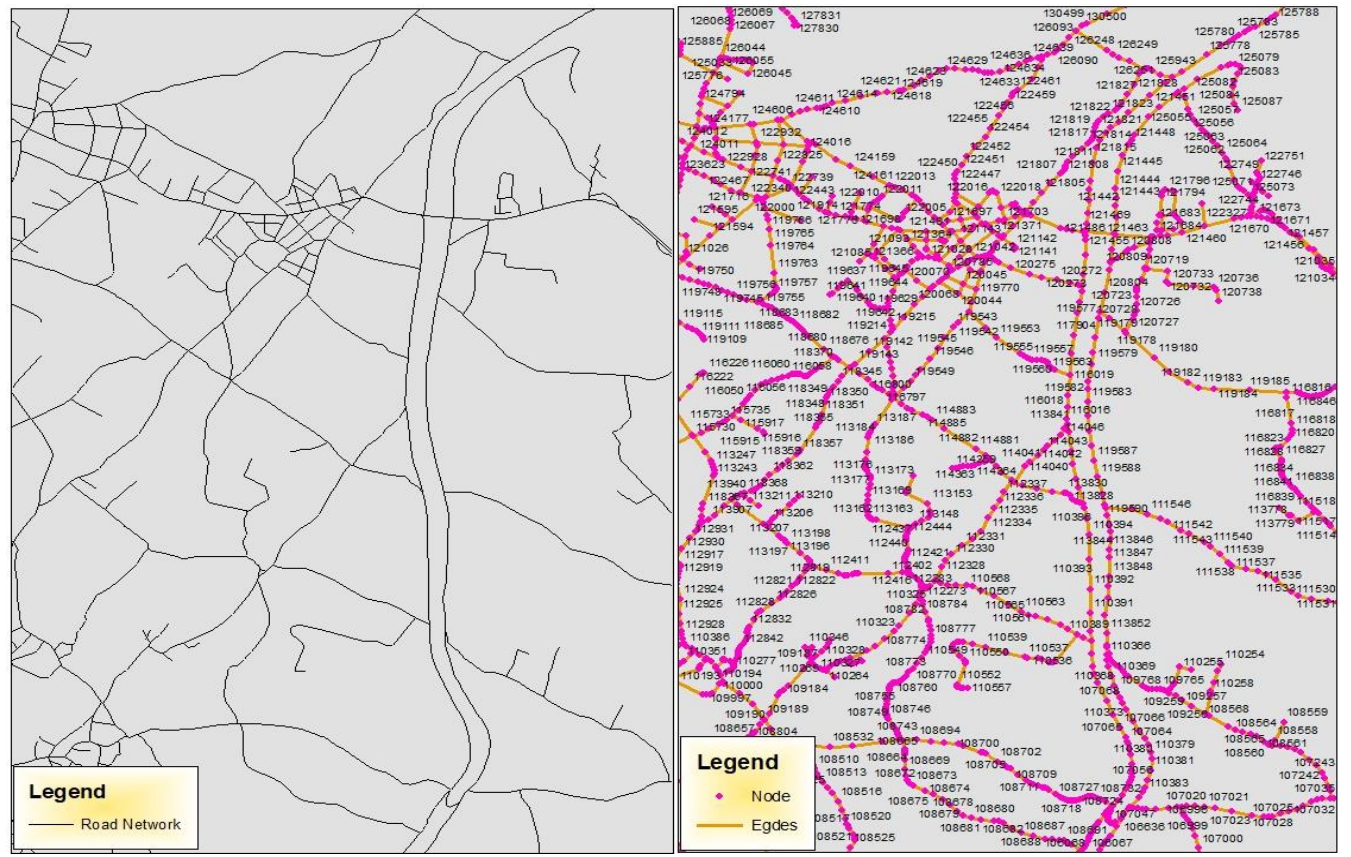

Figure 1. Transformation of the network into nodes - edges. Nodes and edges include additional spatial information such as altitude and proximity to POIs. The proximity is the Euclidean distance between all nodes of spatial network and POI of study area.

Table 2. Spatial data used in spatial network analysis

\begin{tabular}{cccc} 
Gid & Source & Target & Cost (distance in meters) \\
\hline 1 & 1 & 2 & 26,93 \\
2 & 2 & 3 & 17,6 \\
3 & 3 & 4 & 21,66 \\
4 & 4 & 5 & 13,02 \\
5 & 5 & 6 & 16,42 \\
6 & 6 & 7 & 30,42 \\
7 & 7 & 8 & 21,36 \\
8 & 8 & 9 & 21,7 \\
9 & 9 & 10 & 15,56 \\
\hline
\end{tabular}

The distance metric depicted in Table 2, represents the "connection" between nodes of the network in tabular format. It informs about the relationship between nodes as well as the "cost" of this relationship. In this case study the "cost" reflects spatial distance across the network. 
This relationship between nodes could be any other arbitrary number reflecting: distance, costs, time or even effort.

Table 3. Distance matrix between POls and nodes. Nodes include information about altitude

\begin{tabular}{ccccccc} 
Id & $\begin{array}{c}\text { Altitud } \\
\mathbf{e}\end{array}$ & $\begin{array}{c}\text { Distance in } \\
\text { the cultural } \\
\text { sites }(\mathbf{m})\end{array}$ & $\begin{array}{c}\text { Distance in } \\
\text { the tourism } \\
\text { business }(\mathbf{m})\end{array}$ & $\begin{array}{c}\text { Distance in the } \\
\text { settlements }(\mathbf{m})\end{array}$ & X Coords & Y Coords \\
\hline 3 & 28 & 244 & 1258 & 982 & 709136,57 & 4315239,33 \\
4 & 20 & 228 & 1251 & 985 & 709130,94 & 4315260,26 \\
5 & 13 & 222 & 1252 & 983 & 709131,76 & 4315273,25 \\
6 & 20 & 218 & 1255 & 977 & 709135,99 & 4315289,11 \\
7 & 20 & 209 & 1257 & 9 & 709137,51 & 4315319,5 \\
8 & 14 & 207 & 1260 & 970 & 709140,44 & 4315340,66 \\
9 & 10 & 207 & 1264 & 966 & 709144,06 & 4315362,05 \\
10 & 10 & 213 & 1271 & 959 & 709150,19 & 4315376,36 \\
11 & 10 & 220 & 1279 & 953 & 709157,11 & 4315387,19 \\
\hline
\end{tabular}

The use of the proposed spatial algorithm is having as inputs the geographical elements of the geodatabase identified paths based on characteristics of the network and the broader geographical area, which were grouped in the form of spatial filtering criteria. The selection of a suitable path in the network requires the sequential execution of a number of "filtering queries" such as the ones shown below in Table 4. During the execution of a spatial query, the algorithm returns some candidate results. The sequential use of these spatial filters eliminates possible solutions and at the end after the 6th spatial query, we end up with a single solution which fulfils all 6 of the queries simultaneously.

Table 4. Filtering queries (with spatial criteria) executed for the identification of a suitable result.

Spatial Queries

Candidate Results Found

\begin{tabular}{llc}
\hline 1 & Total length of $41 \mathrm{~km}$ (with a deviation of $+-1000 \mathrm{~m}$ ) & 2500 \\
2 & Find ID<1500 with Total length of $41 \mathrm{~km}$ & 2000 \\
3 & Start-End altitude diff of $60 \mathrm{~m}$ & 1400 \\
4 & Find paths with mean distance in the settlements & 908 \\
5 & Find paths with mean distance in the cultural sites & 67 \\
6 & Find paths with mean distance in the tourism business & 1 \\
\hline
\end{tabular}

The algorithm has identified one (1) acceptable candidate path in the spatial network of Lesvos Island (Figure 2). This fulfills the geometrical characteristics used as inputs in the algorithm. Each spatial criterion that was set in the search algorithm minimized the possible candidate paths. This single suitable network path was identified and depicted in the following map. 


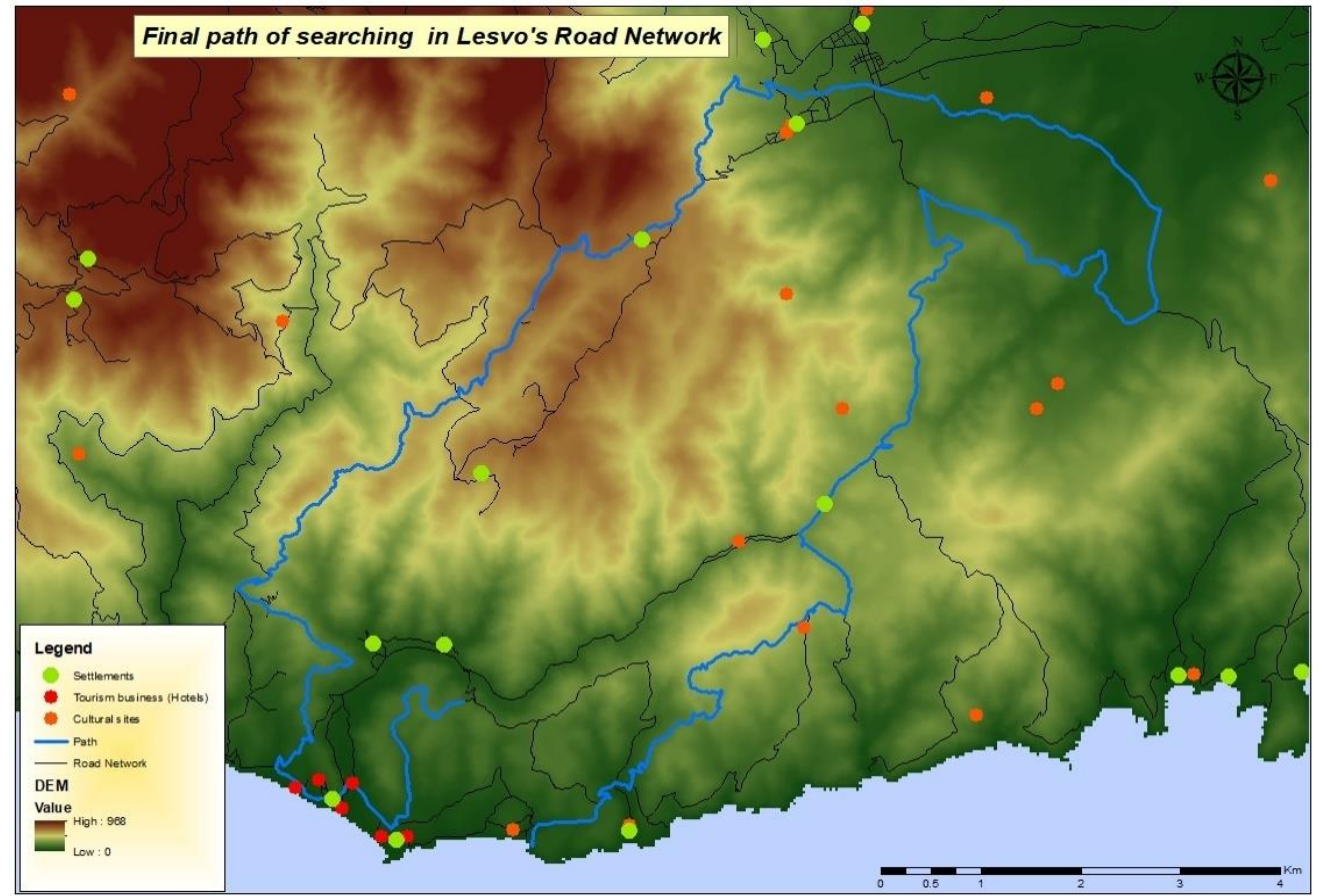

Figure 2. Acceptable path in the road network of Lesvos Island after searching in spatial database using spatial criteria

There are many similarities between spatial criteria and the final solution. For example, the different altitude between starting and ending points in the final path are similar with criterion 3 (figure 4). Also the path's total length is $40000 \mathrm{~m}$, having the mean distance from settlements, cultural monuments and tourism business (figure 3). Specifically, the solution path with code name "monopati 13" is described by 1846 nodes which contains spatial information about total length (Spatial Queries1-2) and the altitude from the start node to its end node. The mean altitude of the route is $179.4924 \mathrm{~m}$ and the altitude difference of the start-end node is up to $60 \mathrm{~m}$ (Spatial Queries3). From the total distance between the nodes of the spatial network and the points of interest was calculated mean distance to settlements (Spatial Queries4), mean distance to cultural sites (Spatial Queries5) and mean distance to tourism business (Spatial Queries6) in the study area.

\begin{tabular}{|l|l|l}
\hline Name & Type & Value \\
apantisi2 & list [1] & List of length 1 \\
II1]] & list [12] & List of length 12 \\
onoma & character [1] & 'Monopati 13: 1 -> 311' \\
MeanAlt & double [1] & 179.4924 \\
AllAltitudes & double [1846] & $262028201320 \ldots$ \\
allIDS & double [1846] & $123456 \ldots$ \\
StartNode & double [1] & 1 \\
EndNode & double [1] & 311 \\
MeanDisttoA & double [1] & 1464.536 \\
meanDisttoB & double [1] & 4502.81 \\
meanDisttoC & double [1] & 3472.14 \\
meanDisttoD & double [1] & 1216.942 \\
StartAltitude & double [1] & 26 \\
EndAltitude & double [1] & 85 \\
\hline
\end{tabular}

Figure 3. Spatial characteristics of the solution path. 
Figure 3 depicts the structural information about the single solution. It consists of information about the geometrical characteristics of the path as well as the average distance of the path to the closest POls.

The following profile-plot depicts the altitude characteristics of a single solution (a path in the network). The horizontal axes depicts the ID of the node, and the vertical the altitude (above sea level) of each node. The spatial distance between each node in the horizontal axes is 7 meters on average. So the result plot seems very steep, but it is not so steep actually. For illustration purposes we have brought the nodes close together in the horizontal axes in order to show the overall altitude profile of the solution path.

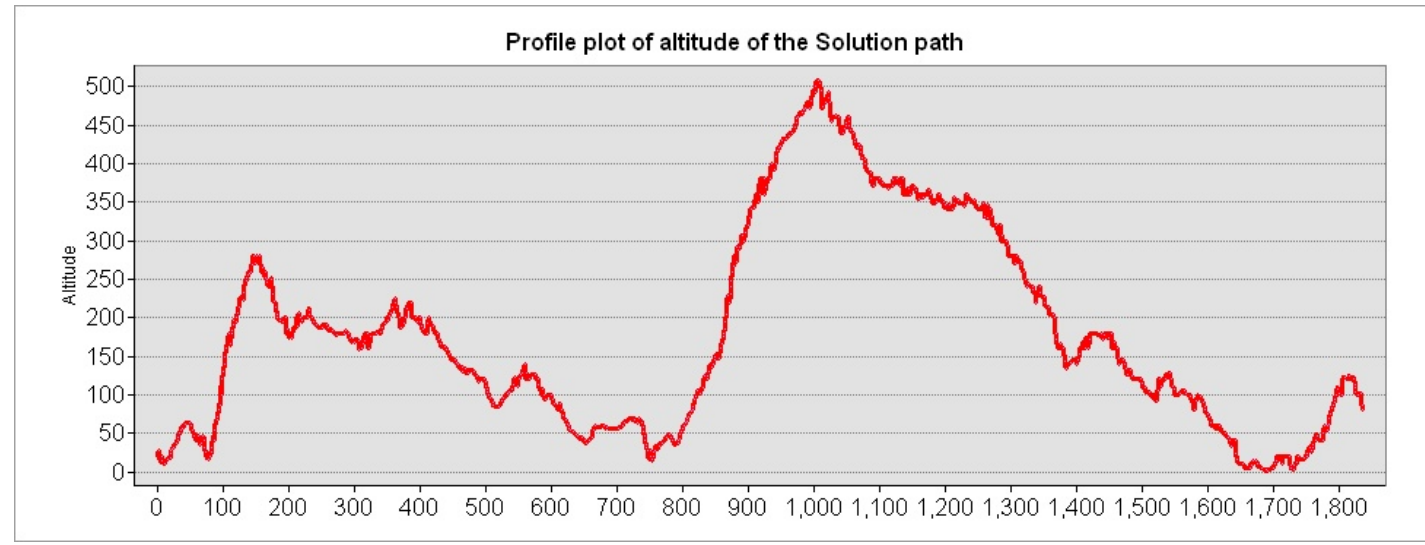

Figure 4. The Profile-plot of altitude of the solution path is presented the altitude difference of the start-end node (up to $60 \mathrm{~m}$ ). The result is similar with spatial query 3

The use of large number of nodes, edges and POls with spatial information is an important factor in identifying a suitable candidate final route (solution) that meets the spatial criteria. The size of the initial dataset (nodes, edges) has a direct influence on the ability of the algorithm to identify a single suitable solution as it increases the algorithm flexibility in the "search space".

Also, the sequence of spatial queries contribute significantly to the results as changes in the sequence of search queries may lead to a different path. Usually, the sequence of queries follows the importance of each characteristic. This is, if total length is the most important aspect of our spatialsearch then this query will be executed first. Then, less important queries will be executed last.

Lastly, the interoperability of an open source programming language such as $\mathrm{R}$ programming language and the Postgresql spatial database ensures the immediate formulation of spatial queries, as well as rapid extraction of acceptable results as well as the interoperability of the algorithm. The use of $\mathrm{R}$ programming language helps towards the interconnection of the algorithm with modern (state of the art) systems for further development and implementation.

\section{DISCUSSION}

Spatial entities in combination with geoinformatics tools and methods are main parts in policy planning and decision-making in addressing spatial problems. The aim is to contribute to an integrated spatial planning that aims at sustainable development at all spatial scales. Spatial network has been viewed as a major dynamic force in influencing quality of life and shaping regions all over the globe.

In this work, our primary goal is to combine many variables with spatial statistics methods in order to develop a mathematical algorithm for identifying "similar" paths in spatial networks using geoinformatics technologies and spatial databases. The results of this work show that the identification of a network route in a spatial network can deviate from traditional methods (Dijkstra's shortest path, "Travelling Salesman Problem") and incorporate some weighted subfactors of a geographical nature such as: altitude of nodes, geographical distance at the edges and the distance between POls and spatial network of the study area. This expands the potential use of the algorithm, making it suitable for planning and decision making applications. 
Thus the present work fills a scientific gap in the international literature on spatial network analysis, creating innovate methods and tools of analysis take into account not only the geometric (topology) but also the geographical aspects (topography) of spatial networks.

The algorithm has been developed in an open source programming language ( $R$ library), which will be freely available for use and will serve as a supportive decision-making tool for solving spatial network problems. The algorithm can be also applied to other scientific fields focusing on spatial networks such as transport, finding emergency routes (Derekenaris et al., 2001), energy (Feldman et al., 1995), environment (Lehner et al., 2013).

Regarding sports activities, identifying an athletic route, athletes have the ability to improve their preparation, as they are practicing on a route similar to the one they will use at the athletic event. It also reduces their transportation by saving resources, while reducing the difficulties in frequent visits to the venue (Kavroudakis et al., 2019). It should be noted that the creation of these routes may also contribute to local development, as it may function as a tourism development tool, since many athletes can visit the proposed area for training. A good training route which may be very similar to high level athletic events such as for example the Classic Marathon of Athens may attract athletic teams for training. This may have a direct impact on local business and touristic activities in the area and help towards the fulfilment of local development goals.

All the above are confirmed by the findings of the case study and the creation of a route using criteria similar to those of the classic marathon of Athens. Criteria such as distance, altitude difference of the start and finish point combining with points of interest gave a path similar in many parts with target path.

However, open source software was important for finding the final path as they contributed to the creation of new tools and methods for analyzing spatial networks. The Postgresql database I processed in a relatively short period of time the road network of the whole of Lesvos, checking all the topological relations of nodes and edges. Also the ability to calculate the proximity of the network from points of interest created a table of spatial characteristics quickly accessible for the application of spatial criteria. Also important was the use of the $R$ programming language as it enabled the creation of a new and innovative algorithm for searching for optimal paths within a spatial network by making accurate and fast mathematical calculations in the database of both topological and geographical character. In this way, significant limitations of the standard commercial GIS packages were overcome as they did not include the appropriate plugins for the analysis of spatial networks.

A potential further expansion of this work could be the incorporation of a set of multivariate indices for the identification of "similarity" of paths in spatial networks. This multivariate aspect of the algorithm can include weighted approaches in identifying suitability of paths or may include weighted ranking of the POls during the pre-processing phase.

In this way we will be able to solve spatial problems in areas with similar spatial networks, such as traffic jams in urban areas and the reduction of road accidents (Vaitis et al., 2019) in networks that present maintenance and construction problems.

\section{CONCLUSIONS}

Describing spatial networks as a synthesis of topological data derived from the scientific field of Graph Theory and spatial relationships created through GIS, an algorithm was developed for the smart querying optimal paths of a road network in a Spatial Database. The proposed algorithm of this work is based on spatial criteria highlighting additional features such as social, cultural and environmental characteristics of the island of Lesvos. These characteristics represent the categorization of the POls together with geographical values of the spatial network such as altitude, aspect, slope etc.

The algorithm was developed with the help of open-source geoinformatics software such as the $\mathrm{R}$ programming language, giving valid and reliable results through spatial queries performed in a spatial database in Postgresql (Postgis-Pgrouting) environment. The spatial 
database contained spatial information about nodes and network edges such as altitudes and distances from points of interest.

The methodology and the search algorithm can be applied to any Spatial Network (transportations, environment, energy) described by its geographical features, considering all kinds of geographical issues. This may also include abstract representations of networks such as ancient trading routes or even intercontinental transportation connections. The algorithm can be used in any network dataset and incorporate external points (POIs) in order to assign extra information to the nodes of a network.

The unique scientific contribution of this work is based on the presentation of a new method of spatial analysis based on the combination and interoperability of open source geoinformatics tools ( $R$, Postgresql), enabling the rapid analysis of different types of open data through a variety of spatial algorithms. It also depicts the unique way of approaching spatial networks and applying external geographical features to network nodes in order to be used later for identification of network paths. Also free access to the data and source code used allows to incorporate additional advances in theoretical perspectives and analytical methods, thus facilitating interdisciplinary collaboration of spatial science and education (Ye, X., 2018).

In addition, the results of the processing and visualization of large geospatial data structures will be used to implement alternative local development approaches in areas such as sports and tourism. For example, the use of this algorithm can help athletes in identifying "similar" network routes for training and experimentation while practicing for athletic events. Also, it can help local planners in planning specialized "routes" that fulfil proximity criteria for POls in a city, in order to attract athletes and spectators who will contribute to the promotion and development of each region.

\section{REFERENCES}

Agourogiannis P., Batsaris M. (2019). "Web based Geographical Information System for cultural routes", 2nd International Conference on Cultural Informatics, Communication \& Media Studies 13-15 June, Mytilini.

Ahmadzai, F., Rao, K.M.L., Ulfat, S. (2019). Assessment and modelling of urban road networks using Integrated Graph of Natural Road Network (a GIS-based approach). Journal of Urban Management 8, 109-125. https://doi.org/10.1016/j.jum.2018.11.001

Ahmed, S., Ibrahim, R.F., Hefny, H.A., n.d. GIS-Based Network Analysis for the Roads Network of the Greater Cairo Area 10.

Becker, R. A., Chambers, J. M., and Wilks, A. R. (1988). The New SS Language. Chapman \& Hall, London. [2, 37]

CRAN. (2019). "The Comprehensive R Archive Network.", 2019. http://cran.r-project.org/

CRAN. (2021). "The Comprehensive R Archive Network.", 2021. https://cran.rproject.org/web/packages/dplyr/index.html

Chen, Q., Xu, N. (2019). Research on the Shortest Path Analysis Method in Complex Traffic Environment Based on GIS, in: 2019 IEEE 4th Advanced Information Technology, Electronic and Automation Control Conference (IAEAC). Presented at the 2019 IEEE 4th Advanced Information Technology, Electronic and Automation Control Conference (IAEAC), IEEE, Chengdu, China, pp. 208-212. https://doi.org/10.1109/IAEAC47372.2019.8997883

Derekenaris, G., Garofalakis, J., Makris, C., Prentzas, J., Sioutas, S., Tsakalidis, A. (2001). Integrating GIS, GPS and GSM technologies for the effective management of ambulances. Computers, Environment and Urban Systems 25, 267-278. https://doi.org/10.1016/S0198-9715(00)00025-9 
Dijkstra, E.W. (1959). A note on two problems in connexion with graphs. Numerische Mathematik 1, 269-271. https://doi.org/10.1007/BF01386390

Elsheikh, R.F.A., Elhag, A., Sideeg, S.E.K., Mohammed, A.E., Gism, N.A., Allah, M.S.A. (2016). Route Network Analysis in Khartoum City 17, 8.

Feldman, S.C., Pelletier, R.E., Walser, E., Smoot, J.C., Ahl, D. (1995). A prototype for pipeline routing using remotely sensed data and geographic information system analysis. Remote Sensing of Environment 53, 123-131. https://doi.org/10.1016/0034-4257(95)00047-5

Fischer, M.M. (2006). Spatial analysis and geocomputation: selected essays. Springer, Berlin ; New York.

Fischer, M.M., Nijkamp, P. (1992). Geographic information systems and spatial analysis. Ann Reg Sci 26, 3-17. https://doi.org/10.1007/BF01581477

Hu, H., Wang, F. (2016). Analysis and Application of the Shortest Path Algorithm based on Geographic Information System. International Journal of Earth Sciences and Engineering 09,6 .

Kavroudakis D., Agourogiannis P., Batsaris M. Vaitis M., Kavroudakis E., Kouloumentas P. (2019) Similarity of paths in spatial networks: The case of long-distance athletic events, 22ndAGILE Conference, June 2019, Limassol, Cyprus

Kuby, M., Lim, S. (2007). Location of Alternative-Fuel Stations Using the Flow-Refueling Location Model and Dispersion of Candidate Sites on Arcs. Networks and Spatial Economics 7, 129-152.

Lehner, B., Grill, G. (2013). Global river hydrography and network routing: baseline data and new approaches to study the world's large river systems: GLOBAL RIVER HYDROGRAPHY AND NETWORK ROUTING. Hydrological Processes 27, 2171-2186. https://doi.org/10.1002/hyp.9740

Lu, B., Sun, H., Harris, P., Xu, M., Charlton, M. (2018). Shp2graph: Tools to Convert a Spatial Network into an Igraph Graph in R. ISPRS International Journal of Geo-Information 7, 293. https://doi.org/10.3390/ijgi7080293

Malczewski, J., 2006. GIS-based multicriteria decision analysis: a survey of the literature. International Journal of Geographical Information Science 20, 703-726. https://doi.org/10.1080/13658810600661508

Panahi, S., Delavar, M.R. (2008). A GIS-based Dynamic Shortest Path Determination in Emergency Vehicles 7.

Paraskevopoulos, Y., Bardosa, A., Photis, Y.N. (2019). EXPLORING THE IMPACT OF NETWORK CONFIGURATION AND TRANSPORT ACCESSIBILITY ON POPULATION DYNAMICS. THE CASE OF NAXOS ISLAND, GREECE 10, 18.

PgRouting Community. Pgrouting 2.3. (2019). https://pgrouting.org/index.html

PostGIS contributors PostGIS Manual (2019)

Rodrigue, J.-P., Comtois, C., Slack, B. (2013). The geography of transport systems, Third edition. ed. Routledge, London; New York.

Shang, S., Chen, L., Wei, Z.-W., Guo, D.-H., Wen, J.-R. (2016). Dynamic Shortest Path Monitoring in Spatial Networks. Journal of Computer Science and Technology 31, 637648. https://doi.org/10.1007/s11390-016-1653-3 
Tobler, W.R. (1970). A Computer Movie Simulating Urban Growth in the Detroit Region. Economic Geography 46, 234. https://doi.org/10.2307/143141

Vaitis M., Kavroudakis D., Koukourouvli N., Simos D. (2017). Spatiotemporal Analysis of road traffic accidents at Lesvos island Greece, Smart, Inclusive and Resilient Small and Medium-sized Cities and Island Communities in the Mediterranean: Exploring Current Research Paths and Experience-based Evidence, Heraklion-Crete / Greece, September 28-29, 2017

Ye, X. (2018). Open Data and Open Source GIS, in: Comprehensive Geographic Information Systems. Elsevier, pp. 42-49. https://doi.org/10.1016/B978-0-12-409548-9.09592-0

Yu, C., Lee, J., Munro-Stasiuk, M.J. (2003). Research Article: Extensions to least-cost path algorithms for roadway planning. International Journal of Geographical Information Science 17, 361-376. https://doi.org/10.1080/1365881031000072645

Zeng, W., Church, R.L. (2009). Finding shortest paths on real road networks: the case for $A^{*}$. International Journal of Geographical Information Science 23, 531-543. https://doi.org/10.1080/13658810801949850 